\title{
Plasma modes in layered superconductors
}

\author{
I.N. Askerzade ${ }^{\mathrm{a}, \mathrm{b}, *}$, B. Tanatar ${ }^{\mathrm{c}}$ \\ a Department of Physics, Faculty of Sciences, Ankara University, Tandogan-Ankara 06100, Turkey \\ ${ }^{\mathrm{b}}$ Institute of Physics, Azerbaijan National Academy of Sciences, Baku-AZ1143, Azerbaijan \\ ${ }^{\mathrm{c}}$ Physics Department, Bilkent University, Bilkent, Ankara, Turkey
}

Received 8 October 2004; received in revised form 15 December 2004; accepted 27 December 2004

\begin{abstract}
An expression for the plasmon spectrum in the layered superconductors with arbitrary thickness of planes, which varies within a wide range is obtained. The obtained result can be attractive for the explanation of experimental data on plasmon modes in cuprates and other recently discovered superconductors.
\end{abstract}

(C) 2005 Elsevier B.V. All rights reserved.

PACS: 74.80.Dm; 74.20.Mn

\section{Introduction}

Until to discovery of cuprate superconductors, it was generally accepted [1] that plasma modes could not exist below the superconducting gap. In superconductors, the Coulomb interaction shifts all the density oscillation modes to the plasma frequency $[2,3]$ thus at an energy much higher than the superconducting gap. An exception to this rule is the layered superconductors, whose penetration depth is very large along the $c$-axis

\footnotetext{
* Corresponding author. Tel.: +90 312 2126720; fax: +90 312 2232395.

E-mail address: iasker@science.ankara.edu.tr (I.N. Askerzade).
}

(perpendicular to the $\mathrm{CuO}_{2}$ planes) and the static dielectric constant along the $c$-axis is estimated very large. Due to these factors the plasma frequency along $c$-axis is strongly reduced. Plasma oscillations of superconducting electrons was observed in HTSC crytals [4-7].

It is well known, that HTSC has confirmed once again that variation of the structure anisotropy strongly affects the physical properties. In these materials the number of superconducting planes $\mathrm{CuO}_{2}$ per unit cell $[8,9]$, as well as the distance between the planes are changed with variation of the dopant concentration or oxygen deficiency. Recent development in epitaxial technology allows to grow artifical SC/dielectric superlattices [10,11]. Variation of the superlattice parameters strongly 
influence the plasmon dispersion relations. Although many theoretical work have discussed plasma oscillations in cuprate superconductors [12], the explicit formula for the dependence of the plasma frequency on the thickness of conducting layer has not appeared. Therefore, finding the dependence of the plasmon spectrum on the thickness of SC and dielectric layers becomes an interesting problem. In this note we will study plasma spectrum of layered superconductors with arbitrary layer thickness.

\section{Theoretical background}

The plasmon modes can be determined as poles of the Dyson's equation for Coulomb potential, which has a form [13]:

$$
V\left(\mathbf{q}, q_{z} ; \omega\right)=\frac{V\left(\mathbf{q}, q_{z}\right)}{\left(1+V\left(\mathbf{q}, q_{z}\right) \Pi\left(\mathbf{q}, q_{z} ; \omega\right)\right)},
$$

where $V\left(\mathbf{q}, q_{z}\right)$ is the bare Coulomb interaction in a superlattice. The polarization operation operator $\Pi\left(\mathbf{q}, q_{z} ; \omega\right)$ within random-phase-approxiamation(RPA) is given by

$$
\begin{aligned}
& \Pi\left(\mathbf{q}, q_{z} ; \omega\right) \\
& \quad=2 \sum_{\mathbf{p}, p_{z}} \frac{\left(n_{\mathrm{F}}\left(\xi\left(\mathbf{p}+q, p_{z}+q_{z}\right)\right)-n_{\mathrm{F}}\left(\xi\left(\mathbf{p}, p_{z}\right)\right)\right)}{\xi\left(\mathbf{p}, p_{z}\right)-\xi\left(\mathbf{p}+\mathbf{q}, p_{z}+q_{z}\right)+\omega+i 0^{+}},
\end{aligned}
$$

where $n_{\mathrm{F}}(x)$ is the Fermi distribution function. The electron spectrum for layered superconductors $\xi\left(\mathbf{p}, p_{z}\right)$ is defined by the formula

$$
\xi\left(\mathbf{p}, p_{z}\right)=\frac{p^{2}}{2 m}+2 t\left(1-\cos p_{z} D\right)-E_{\mathrm{F}} ; \quad D=a+d
$$

where $\mathbf{p}$ is the two dimensional momentum vector inside a conducting layer, $p_{z}$ is the $z$ component of the momentum vector; $E_{\mathrm{F}}=\frac{p_{\mathrm{F}}^{2}}{2 m}$ is the Fermi energy $t$ is the interlayer tunneling integral, $d$ is the SC layer thickness and $a$ is the distance between these layers. The geometry of the layered structures we consider in this work is depicted in Fig. 1. $t$ characterizes the intensity of electron tunneling between the layers and must depend on the ratio $a / b$ as $t=F(a / b)$, where $b$ is the a characteristic dis-

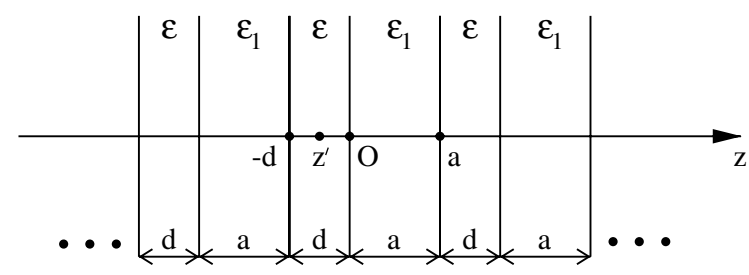

Fig. 1. Geometry of considered system.

tance of the order of unit cell size in the SC layers. The $F(a / b)$ function rapidly decreases when the distance $a$ increases. It is possible, in principle, to obtain an explicit expression for this function provided that the electron density distribution inside the SC layers is known. It is well known, that Josephson coupling between superconducting sheets is proportional to $t^{2}$ [14]. On the other hand, dimensionless parameter $l$, which characterize the strength of Josephson coupling between superconducting layers is determined by the transmission coefficient of tunnel barrier $D(x)$. At small $D(x)$, parameter $l$ is given by expression [15]

$l=\frac{7 \zeta(3) \xi_{0}}{3 \pi^{2}}\left[\int_{0}^{1} x D(x) \mathrm{d} x\right]^{-1}$,

and at high $D(x)$ by the expression

$l=\frac{\pi^{4} \xi_{0}}{28 \zeta(3)}\left[\int_{0}^{1} x^{3}(1-D(x)) \mathrm{d} x\right]$,

where $\zeta(3)$ is the Riemann function, $\xi_{0}$ is the coherence length, $x=\cos \phi, \phi$ is the incidence angle of electrons to the boundary superconductordielectric. The single-particle energy spectrum for the carriers given in Eq. (3) was previously used in several works [16] (see also Ref. therein), $[17,18]$ for the calculation of various properties in layered superconductors.

To calculate plasmon spectrum, we use the expression for the bare Coulomb interaction $V\left(q, q_{z}\right)[19]$ of charged particles in a periodic layered system, consisting of alternating layers with different values of dielectric constant in the large wavelength approximation:

$$
\begin{aligned}
V(n(a+d), q)= & \frac{2 \pi \mathrm{e}^{2}}{\epsilon_{1} q} \exp \left(-n q_{0}\right) \\
& \times \frac{\left(1+\gamma^{+}\right)\left(1-\gamma^{-}\right)}{\left(\gamma^{-}-\gamma^{+}\right)},
\end{aligned}
$$


where

$$
\begin{aligned}
& \gamma^{(+,-)} \\
& =\frac{\left.\exp (q a)-\exp \left((+,-) k_{0}\right)\right)(\alpha \exp (-q d)+\beta \exp (q d))}{\exp (-q a)-\exp \left((+,-) k_{0}\right)(\beta \exp (-q d)+\alpha \exp (q d))},
\end{aligned}
$$

$$
\begin{aligned}
k_{0}= & \cos h^{-1}(\cos h q(a-d) \\
& \left.+\frac{2 \alpha^{2}}{(2 \alpha-1)} \sin h(q a) \sin h(q d)\right)
\end{aligned}
$$

In Eqs. (5) and (6), the following definitions are introduced: $\alpha=\frac{(1+\eta)}{2}, \beta=\frac{(1-\eta)}{2}$, where $\eta=\frac{\epsilon_{1}}{\epsilon}$ with $\epsilon$ and $\epsilon_{1}$ being the dielectric constant of a conducting layer and dielectric, respectively in the case of HTSC (see Fig. 1). Using the following expression for the Fourier transformation [20]

$\sum_{n} \exp \left(-n q_{0}\right) \exp \left(-i n q_{z}(a+d)\right)=\frac{\sin h q_{0}}{\cos h q_{0}-\cos q_{z}(a+d)}$,

we finally obtain:
In the case $a \gg d$ the Coulomb potential presented by Eq. (8) reduces to the following expression:

$V\left(q, q_{z}\right)=\frac{2 \pi \mathrm{e}^{2}}{\epsilon_{1} q} \frac{s h q a}{c h q a-\cos q_{z} a}$

In the opposite asymptotic case of $a \ll d$ Eq. (8) for $V\left(q, q_{z}\right)$ is obtained by the replacement $\epsilon_{1} \rightarrow \epsilon$ and $a \rightarrow d$ are employed. In Fig. 2 we plot dependence of $V\left(q, q_{z}\right) / \frac{2 \pi \mathrm{e}}{\epsilon q}$ on the parameter $d l a$ for different values of $\eta=\frac{\epsilon_{1}}{\epsilon}$ with $q_{z} a=q a=1$. It is clear that as the thickness of conducting layer increases the Coulomb repulsion is decreased. Using Eqs. (1), (4) and (8) we can obtain the final expression for the plasmon spectrum in layered superconductors as

$\omega^{2}\left(q, q_{z}\right)=\left(v_{\mathrm{F}}^{2} q^{2}+8 t^{2} \sin ^{2} \frac{\left(q_{z} D\right)}{2}\right) \Pi(0) V\left(q, q_{z}\right)$,

where $v_{\mathrm{F}}$ is the velocity of electrons on the Fermi surface. At $t=0 \quad q_{z} \rightarrow 0$ and $q D \gg 1$ we obtain the spectrum of two-dimensional plasmons in long wavelength approximation:

$V\left(q, q_{z}\right)=\frac{2 \pi \mathrm{e}^{2}}{\epsilon q} \frac{\alpha \sinh (q(a+d))+\beta \sinh (q(a-d))}{\alpha^{2} \cos h(q(a+d))-\beta^{2} \cosh (q(a-d))-\eta \cos \left(q_{z}(a+d)\right)}$

It is interesting to discuss question related with conditions in which several atomic layers can be approximated by the continuum dielectric medium. It is well known, that at the contact region of different layers in superlattice the crystal structure is deformed and as a result dielectric constant in this region is different from those for the bulk maretial. Due to that, for our purpose of finding the dependence of the plasmon frequency on the thicknesses of conducting and dielectric layers, dielectric constants $\varepsilon$ and $\varepsilon_{1}$ presented here can be considered as effective dielectric contants of layers. In our opinion, introducing more realistic function for the change of dielectric constant (different from square-well-like changing, which was used in [19]), will change our results unconsiderably. Similar questions was discussed by $[21,22]$ many years ago in relation with exitonic superconductivity in "sandwich" structures.

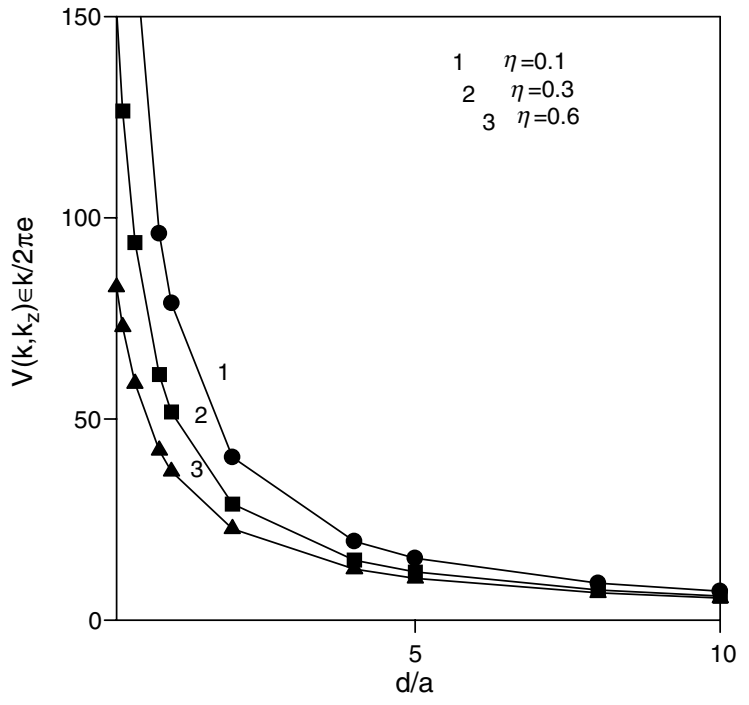

Fig. 2. Dependence of $V\left(q, q_{z}\right) / 2 \pi e^{2} / k$ versus $d / a$. 
$\omega(q)=v_{\mathrm{F}}\left(\frac{2 q}{a_{\mathrm{b}}}\right)^{\frac{1}{2}}$,

where $a_{\mathrm{b}}$ Bor radius for free electron, $a_{\mathrm{b}}=\frac{1}{m \mathrm{e}^{2}}$.

In the case $q D \ll 1$ and $q_{z} D \ll 1$, the plasma frequency depends on the direction of wave vector:

$\omega\left(q, q_{z}\right)=\left(\frac{2}{a_{\mathrm{b}} D}\right)^{\frac{1}{2}}\left[v_{\mathrm{F}}^{2}+\left(t^{2} D^{2}-v_{\mathrm{F}}^{2}\right) \cos ^{2} \theta\right]^{\frac{1}{2}}$,

where $\theta$ is the angle between wave vector and normal vector to the layer. As follows from Eq. (12) the spectrum of plasmons is strongly anisotropic. Frequency of plasma oscillations with wave vector perpendicular to layer is $\frac{t D}{v_{\mathrm{F}}} \ll 1$ times smaller than plasma frequency in the layer.

\section{Results and discussion}

The plasmon frequency for a superlattice is given by the expression in Eq. (12). At long wavelenght $\left(q, q_{z} \rightarrow 0\right)$ we have an optical plasmon mode (bulk plasmon)

$\omega^{2}(0,0)=\frac{8 E_{\mathrm{F}} \mathrm{e}^{2}}{\epsilon} \frac{a+\eta d}{\alpha^{2}(a+d)^{2}-\beta^{2}(a-d)^{2}}$,

In the other limit $q_{z}=\frac{\pi}{D}$, we obtain in the lower branch an acoustic plasmon mode. For the $q_{z}=\frac{\pi}{D}, q(a+D) \ll 1$, and $\frac{t}{E_{\mathrm{F}}} \ll 1$ we obtain

$\omega(q)=\omega(0,0) \frac{\alpha^{2}(a+d)^{2}-\beta^{2}(a-d)^{2}}{2(a+\eta d) \eta} q$,

The plasmon spectrum of a layered superconductors has a rather complicated structure. The plasmon modes for $0<q_{z}<\frac{\pi}{D}$ form a band as shown in Fig. 3. The size of the band is defined by the parameters $\eta$ and ratio $\frac{a}{d}$. It is also important to note that in the limit $q_{z \mathrm{D}} \rightarrow \pi$ the slope of acoustic plasmons $\frac{\mathrm{d} \omega}{\mathrm{d} q}\left(q_{z}=\frac{\Pi}{D}\right)$ is greater than in the case $q_{z}$ $\mathrm{D}=0$. In Fig. 4 we plot the dependence of the normalized slope of acoustic plasmon modes $\frac{\mathrm{d} \omega}{\mathrm{d} q}\left(q_{z}=\frac{\pi}{D}\right)$ versus ratio parameter $d / a$. It is clear that by increasing the thickness of conducting layer, the slope $\frac{\mathrm{d} \omega}{\mathrm{d} q}\left(q_{z}=\frac{\pi}{D}\right)$ is increased. Such conclusion is in good agreement with numerical calculations performed by Pindor and Griffin [23], where a periodic stacks of planes were considered.

We can see that increasing the thickness of metallic layer leads to a decrease in the plasmon

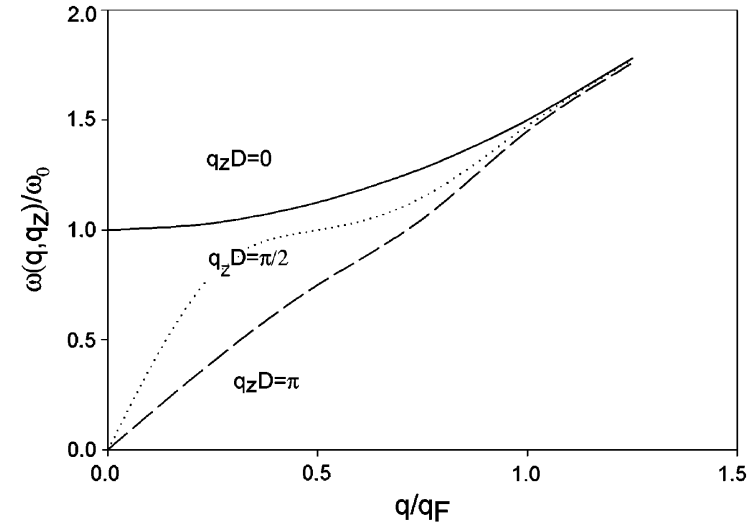

Fig. 3. Plasmon band for layered superconductor, where $\omega_{0}=v_{\mathrm{F}}\left(\frac{2}{a_{\mathrm{b}} D}\right)^{\frac{1}{2}}$.

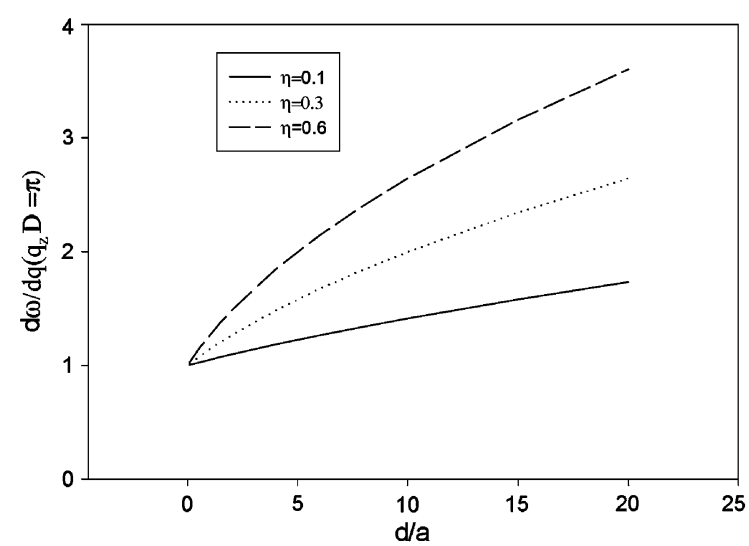

Fig. 4. Dependence of $\frac{\mathrm{d} \omega}{\mathrm{d} q}\left(q_{z}=\frac{\pi}{D}\right)$ versus dla.

frequency $\omega(0,0)$. These results can be useful for the explanation the experimental data for $\mathrm{YBa}$ $\mathrm{CuO}(\omega(0,0)=2.3 \mathrm{eV})$ [24] and $\mathrm{Bi}_{2} \mathrm{Sr}_{2} \mathrm{CaCu}_{2} \mathrm{O}_{8}$ $(\omega(0,0)=1 \mathrm{eV})[25,26]$ compounds. It is well known that in $\mathrm{YBaCuO}$ there are two $\mathrm{CuO}_{2}$ planes, while in $\mathrm{Bi}_{2} \mathrm{Sr}_{2} \mathrm{CaCu}_{2} \mathrm{O}_{8}$ three $\mathrm{CuO}_{2}$ planes and as a result plasmon frequency is decreased. In our model thickness of conducting layer increases by increasing the number of $\mathrm{CuO}_{2}$ planes. Ratio dla for HTSC corresponds to region at Fig. 2, where Coulomb repulsion changes crucially.

Another interesting problem is related with influence of low energy plasmon modes on superconductivity in layered systems. Consequence of the existence of plasmons on the superconductivity 
was discussed by [27]. As shown in this work low energy plasmons can contribute constructively to superconductvity. Bill et al. [27] (see also [28,29]) considered the simplest form (Eq. (9)) for the Coulomb interaction in layered systems with conducting planes with zeroes thickness. The conducting sheets are stacked along $c$-axis and separated by spaces with dielectric constant $\epsilon_{M}$. The electrons moving within the superconducting sheets $(t=0)$. The purpose of the work [27] was to study an increasing influence of the phonon-plasmon interaction on the electron pairing mechanism in framework Eliashberg theory. The plasmon contribution for superconductivity is shown to be dominant in newly discovered layered superconductor metal-intercalated halide nitrides [27].

In more early work [3] was reported study of plasmon modes in layered superconductors with conducting planes with zero thickness using kinetic equations for Green functions. It is shown that at the vicinity $T_{\mathrm{c}}$ plasma oscillations transformed to Carlson-Goldman mode observed in [30]. Unlike other works influence of the order parameter on the plasmon spectrum also was discussed.

In Ref. [23] it was shown that plasmon modes which are expected in cuprate superconductors should be characteristic of a superlattice with a basis of several metallic sheets. Numerical results are given for the superlattice plasmon dispersion relations for two and three sheets/unit cell. Electron gas in metallic sheets is considered as twodimensional. If the spacing of the sheets is small compared to the superlattice period, it is shown that the low-frequency plasmon branch are essentially identical to those of an isolated bilayer or trilayer.

Unlike to the approachs presented above $[3,23,27]$ we have developed simplest model taking into account thickness $d$ of the conducting sheets. The values of $d$ and the thickness of dielectric layer $a$ for different homologous cuprate series are presented in [31]. The ratio $d / a$ for HTSCs increases with increasing number of $\mathrm{CuO}_{2}$ planes in unit cell, which corresponds to the region in Fig. 2, where the Coulomb repulsion changes crucially and means a considerable change in the plasmon frequency of layered SCs induced by changing the number of $\mathrm{CuO}_{2}$ planes. The value of $d / a=2.3$ corresponds to the $\mathrm{Bi}_{2} \mathrm{Sr}_{2} \mathrm{CaCuO}_{8}$ compound. For another cuprate superconductor $\mathrm{YBaCuO}$, the ratio $d / a=1.73$. As noted in [32], for all cuprates, the lattice static dielectric constant varies in the range 6-10, and for $\mathrm{YBaCuO}$, we have a value of about 4 [31]. To estimate $\eta=\frac{\epsilon_{1}}{\epsilon}$, we will use a value of $\epsilon$ in the range 4-10, while $\epsilon_{1}$ can be taken to be about 1. Consequently, $\eta$ varies as $0.1-0.25$.

As a concluding remark, it is interesting to add some considerations on the newly discovered superconductor magnesium diboride [33]. This material also has a layered structure with the boron atoms forming layers of two-dimensional honeycomb lattices (single layer). Recent studies with the growth of single crystals [34] show anisotropy of physical properties in $\mathrm{MgB}_{2}$. Our results can be applied also to $\mathrm{MgB}_{2}$ in the limit, when $d / a$ tends to zero. Calculation of plasma frequency in $\mathrm{MgB}_{2}$, using de Haas van Alphen data, was conducted by [35]. Another pecularity of plasmon modes in $\mathrm{MgB}_{2}$, related with two-band nature of superconductivity in this compound. In this case the appearance of low-energy plasmon branches, so called "demons" [36] appears as a result of two overlapping bands.

In summary, we have shown that with increasing thickness of superconducting layer $\left(\mathrm{CuO}_{2}\right.$ stacks in the case of HTSC) plasma frequency decreases and slope of acoustic plasmon modes $\frac{\mathrm{d} \omega}{\mathrm{d} q}\left(q_{z}=\frac{\pi}{D}\right)$ is increased. Obtained result seems to be attractive from the point of application to HTSC and superconducting superlattices.

\section{Acknowledgments}

I.N.A. thanks Professors H. Yilmaz, B. Unal and S. Atag at Ankara University for their hospitality, Professors F.M. Hashimzade and R.R. Guseinov for useful discussion. B.T. acknowledges support from TUBITAK, TUBA, and NATOSfP. I.N.A. also acknowledges support from NATO reintegration grant (Fel.Rig. 980766).

\section{References}

[1] P.C. Martin, in: R.D. Parks (Ed.), Superconductivity, 1970. 
[2] P.W. Anderson, Phys. Rev. 112 (1958) 1900.

[3] S.N. Artemenko, A.G. Kobelkov, JETP Lett. 58 (1993) 445.

[4] M.M. Doria, F. Parage, O. Buission, Europhys. Lett. 35 (1996) 445

[5] J.H. Kim, H.S. Somal, M.T. Czyzyk, et al., Physica C 247 (1995) 297.

[6] S. Tajima, G.D. Gu, S. Miyamoto, et al., Phys. Rev. B 48 (1993) 16164.

[7] S. Uchida, K. Tamasaku, S. Tajima, Phys. Rev. B 53 (1996) 14558.

[8] C.W. Chu, J. Supercond. 12 (1999) 85.

[9] H. Yamauchi, M. Karppinen, Supercond. Sci. Tech. 13 (2000) R33.

[10] S. Ikegawa et al., Phys. Rev. B 66 (2002) 014536.

[11] H.C. Yang et al., Phys. Rev. B 59 (1999) 8956.

[12] S.L. Drechler, T. Mishonov (Eds.), HTSC and Related Materials, Kluwer Academic, Dordecht, 2001, Chapter 3.

[13] A.A. Abrikosov, L.P. Gorkov, I. Dzyaloshinski, Quantum Field Theoretical Methods in Statistical Physics, Pergamon, New York, 1965.

[14] L.N. Bulaevskii, in: V.L. Ginzburg, D.A. Kirznitz (Eds.), Problems of High-temperature Superconductivity, Nauka, Moscow, 1977.

[15] A.V. Svidzinskii, Space-Inhomogenous Problems of Superconductivity, Nauka, Moscow, 1982.

[16] J. Jiang, J.P. Carbotte, Phys. Rev. B 53 (1996) 12400.

[17] E.P. Nakhmedov, Phys. Rev. B 54 (1996) 6624.

[18] I.N. Askerzade, B. Tanatar, Physica C 384 (2003) 404.

[19] R.R. Guseinov, Phys. Stat. Solidi b. 125 (1984) 257.
[20] I.S. Gradshteiyn, I.M. Rizhik, Tablitsi Integralov, Summ, Ryadov I Proizvedeniy, Izd., Nauka, Moskva, 1963.

[21] G.F. Zharkov, Yu.A. Uspenskii, Zh. Exp. Teor. Fiz. 61 (1971) 2123.

[22] G.F. Zharkov, Yu.A. Uspenskii, Zh. Exp. Teor. Fiz. 65 (1973) 1460.

[23] A. Griffin, A.J. Pindor, Phys. Rev. 39 (1989) 11503.

[24] L.P. Gorkov, N.B. Kopnin, Uspekhi Fizicheskich Nauk 156 (1988) 117.

[25] V.G. Grigoryan, G. Paasch, S.L. Drechsler, Phys. Rev. B 60 (1999) 1340.

[26] L.N. Bulaevski, M.P. Maley, S. Tachiki, Phys. Rev. Lett. 74 (1995) 801.

[27] A. Bill, H. Morawitz, V.Z. Kresin, Phys. Rev. B 68 (2003) 144519.

[28] V.Z. Kresin, Phys. Rev. B 35 (1987) 8716.

[29] V.Z. Kresin, H. Morawitz, Phys. Rev. B 37 (1988) 7854.

[30] R.V. Carlson, A.M. Goldman, Phys. Rev. Lett. 34 (1975) 11.

[31] D.R. Harsmann, A.P. Mills Jr., Phys. Rev. B 45 (1992) 10684.

[32] E.G. Maximov, Usp. Phys. Nauk 170 (2000) 1033.

[33] J. Nagamatsu, N. Nakagawa, T. Muranaka, Y. Zenitani, J. Akimitsu, Nature (London) 410 (2001) 63.

[34] M. Zehetmayer, M. Elstever, H.W. Weber, J. Jun, S.M. Kazakov, J. Karpinski, A. Wisniewski, Phys. Rev. B 66 (2002) 052505.

[35] S. Elgazzar, P.M. Oppeneer, S.L. Drechsler, et al., Solid State Commun. 121 (2002) 99.

[36] J. Ruvalds, Adv. Phys. 30 (1981) 677. 